UCRL-JC-129757

PREPRINT

\title{
Ghost Reflection Analysis for the Main Laser of the National Ignition Facility
}

\author{
R. E. English \\ J. L. Miller \\ J. Schweyen
}

This paper was prepared for submittal to the

Optical Society of America 1998 Summer Topical Meetings

Kailuia-Kona, HI

June 8-12, 1998

June 26, 1998

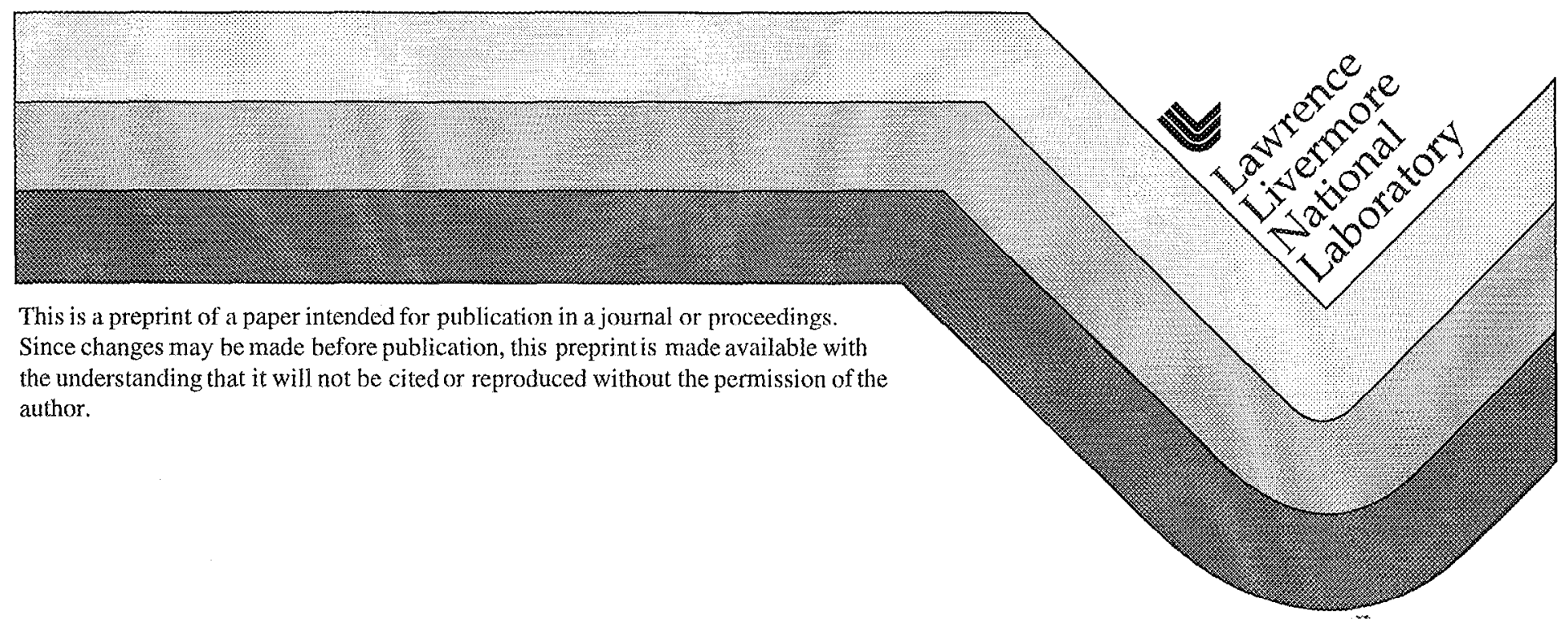


DISCLAIMER

This document was prepared as an account of work sponsored by an agency of the United States Government. Neither the United States Government nor the University of California nor any of their employees, makes any warranty, express or implied, or assumes any legal liability or responsibility for the accuracy, completeness, or usefulness of any information, apparatus, product, or process disclosed, or represents that its use would not infringe privately owned rights. Reference herein to any specific commercial product, process, or service by trade name, trademark, manufacturer, or otherwise, does not necessarily constitute or imply its endorsement, recommendation, or favoring by the United States Government or the University of California. The views and opinions of authors expressed herein do not necessarily state or reflect those of the United States Government or the University of California, and shall not be used for advertising or product endorsement purposes. 


\title{
Ghost reflection analysis for the main laser of the National Ignition Facility
}

\author{
R. Edward English Jr., John L. Miller \\ Lawrence Livermore National Laboratory \\ PO Box 808, L-527 \\ Livermore, CA 94551 \\ (925) 422-3602, (925) 424-6085 fax, english2@llnl.gov \\ John Schweyen \\ Breault Research Organization \\ 6400 East Grant Road, Suite 350 \\ Tucson, AZ 85715 \\ (520) 721-0500, (520) 721-9630 fax
}

\begin{abstract}
Ghost reflections are a major consideration in the optical design of the National Ignition Facility (NIF). The first-order layout (e.g., spacing between components), the lens shape, and the dimensions of the building are strongly affected.

In this paper we will describe the principal ghost reflections that drive the system configuration. Several specific examples will be shown to illustrate how dangerous ghost reflections are avoided and stray light concerns are managed.
\end{abstract}

\section{Introduction}

The NIF comprises several optical subsystems. ${ }^{1}$ The main laser cavity contains 28 optical components, eight of which are AR coated transmissive elements at or near normal incidence to the laser beam; see Fig. 1. Reflections from these surfaces (so-called ghost reflections) are numerous and extensive computation has been required to track them in the NIF optical system.

Because of the high energy and peak power of a NIF laser beam [18kJ (5TW) at the fundamental lasing wavelength, $\lambda=1.053 \mu \mathrm{m}$ ], even small reflections from anti-reflection (AR) coated lenses and windows are significant. A reflection coefficient of $0.1 \%$ yields an $18 \mathrm{~J}$ ghost reflection, a respectable laser in its own right. A reflection from the curved surface of a lens can come to focus, and the irradiance can easily exceed $10^{12} \mathrm{~W} / \mathrm{cm}^{2}$. During operation, we can expect the AR coatings to degrade, and we must plan for reflection coefficients as high as $1 \%$. Thus, in many cases, multiple reflections have been analyzed.

The principal concern arising from ghost reflections is damage to optical components. Focused reflections can easily exceed the optical damage threshold unless the component positions are adjusted so as to avoid the most dangerous ghosts. The ghosts are so numerous in some parts of the system, that precise calculations of locations and tight control of component placement have been necessary. Placement sensitivities are as tight as $2-3 \mathrm{~cm}$ in this laser that is 123 meters long. 


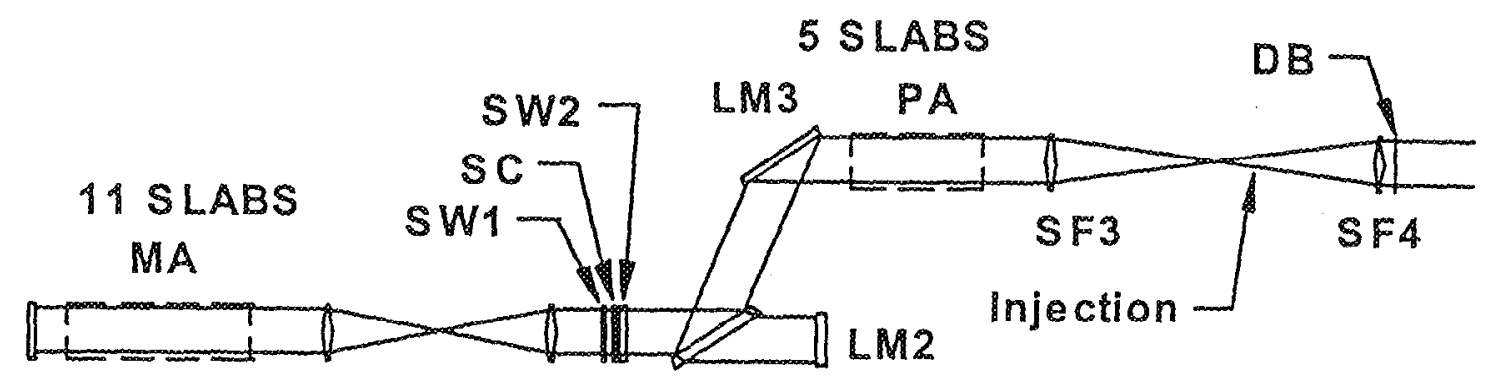

\section{LM1 SF1 SF2 PL}

Fig. 1. The main laser system contains the cavity end mirrors (LM1/2), the main amplifier (MA), the cavity spatial filter lenses (SF1/2), the Pockels cell, which includes two switch windows (SW1/2) and a switch crystal (SC), the polarizer (PL), the elbow mirror (LM3), the power amplifier (PA), the transport spatial filter lenses (SF3/4), and the diagnostic beamsplitter (DB). The pulse is injected at the pinhole plane of the transport spatial filter.

Ghost reflections are primarily controlled by appropriate component spacing and tilts. Ghost stay-out zones are defined near spatial filter lenses. The transport spatial filter lenses are tilted. The Pockels cell components are tilted and wedged to direct spurious reflections to beam dumps or acceptable locations in the spatial filter. The rear surfaces of cavity end mirrors are tilted to direct spurious reflections similarly.

Several methods have been used to analyze dangerous ghost reflections and stray light concerns. First-order analysis used spreadsheets, simplified pictorial representations, and simple fluence calculations. Sequential raytrace models verified first-order analysis, tracked multiple surface bounces, determined precise locations of ghost foci, and analyzed aberrations in ghost reflections. The nonsequential optical models included a whole laser bundle ( 8 beams in $4 \times 2$ configuration), simplified representations of mechanical structures, coating and surface characteristics, and multiple surface bounces with losses and gain. Several examples will be described to illustrate the application of these methods.

\section{Ghost staymout zones for spatial filter lenses}

The spatial filter lenses (SF1, SF2, SF3, SF4 in Fig. 1) are equi-convex. ${ }^{2}$ For a collimated beam incident on an equi-convex lens made, the ghost reflection from the second surface has a focal length $\left(f_{G}\right)$ of

$$
f_{G}=(n-1) /(2 n-1) * f_{S F},
$$

where $n$ is the index of refraction, $f_{G}$ is the ghost focal length, and $f_{S F}$ is the spatial filter lens focal length. For fused silica at $1.053 \mu \mathrm{m}$, the formula reduces to

$$
f_{\mathrm{G}}=0.236 * \mathrm{f}_{\mathrm{ST}}
$$


It should be obvious that the system layout should not place components near single-bounce ghost foci because of the substantial beam energy. After two passes in the cavity, the beam energy is approximately $500 \mathrm{~J}$; after four passes through the main amplifier it is $11 \mathrm{~kJ}$; after the power amplifier it is $18 \mathrm{~kJ}$. For first-order layout, we established a design rule that the ghost stayout zone extended to where the ghost fluence was $10 \%$ of the incident fluence for $0.5 \%$ reflection. ${ }^{\dagger}$ The ghost stay-out length $(\Delta z)$ is

$$
\Delta z=0.289 * \mathrm{f}_{\mathrm{SF}}
$$

The cavity spatial filter lens focal lengths is $11.75 \mathrm{~m}$. The corresponding first-order ghost stay-out zone is about $3.4 \mathrm{~m}$. As seen in Fig. 2, the single-bounce ghost reflection from the spatial filter lens on the last pass in the cavity falls safely in the stay-out zone between the main amplifier slabs and the spatial filter lens.

The transport spatial filter lens focal length is $30 \mathrm{~m}$. The first-order ghost stay-out zone, according to the rule above, would be $8.67 \mathrm{~m}$. Note that the stay-out zone is merely "space" in the laser system. Ultimately, this affects how large the building needs to be or how close together other opto-mechanical assemblies need to be. To reduce the cost of the facility, we decided to tilt the input transport spatial filter lens to reduce the ghost stay-out zone. This complicated the optical design and fabrication because the tilted lens must have a bilaterally symmetric asphere to correct for the third-order coma and astigmatism introduced by the tilt. ${ }^{2}$ The system trade-off was deemed worthwhile, and the ghost stay-out zone for the transport spatial filter (TSF) lens was set to

$$
\Delta Z_{\mathrm{TSF}}=0.222 * \mathrm{f}_{\mathrm{TSF}}=6.66 \mathrm{~m}
$$

\section{Ghost stay-out zone}

\section{LM1 MA}

SF 1

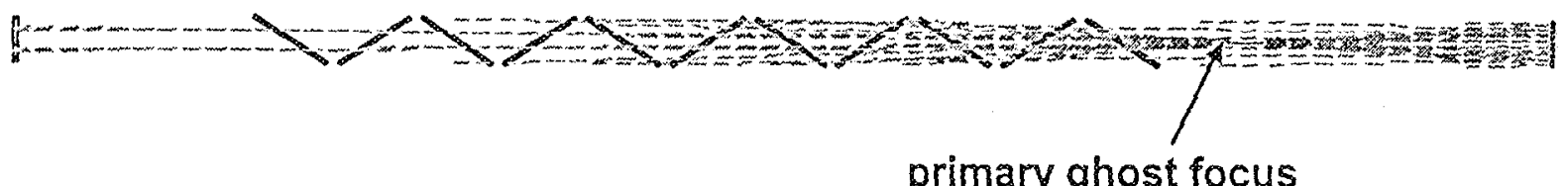

Fig. 2. Pass 2, traveling from left-to-right, impinges on SF1; ghost reflections are generated at both surfaces of the lens. The reflection from side 1 is diverging; the reflection from side 2 is converging and comes to focus in the ghost stay-out as shown in figure.

\footnotetext{
"This is somewhat conservative because the lens damage threshold is set by the full beam energy (i.e., last pass), not the incident beam energy. During later detailed design calculations, the rule was modified to be $10 \%$ of the maximum fluence.
} 


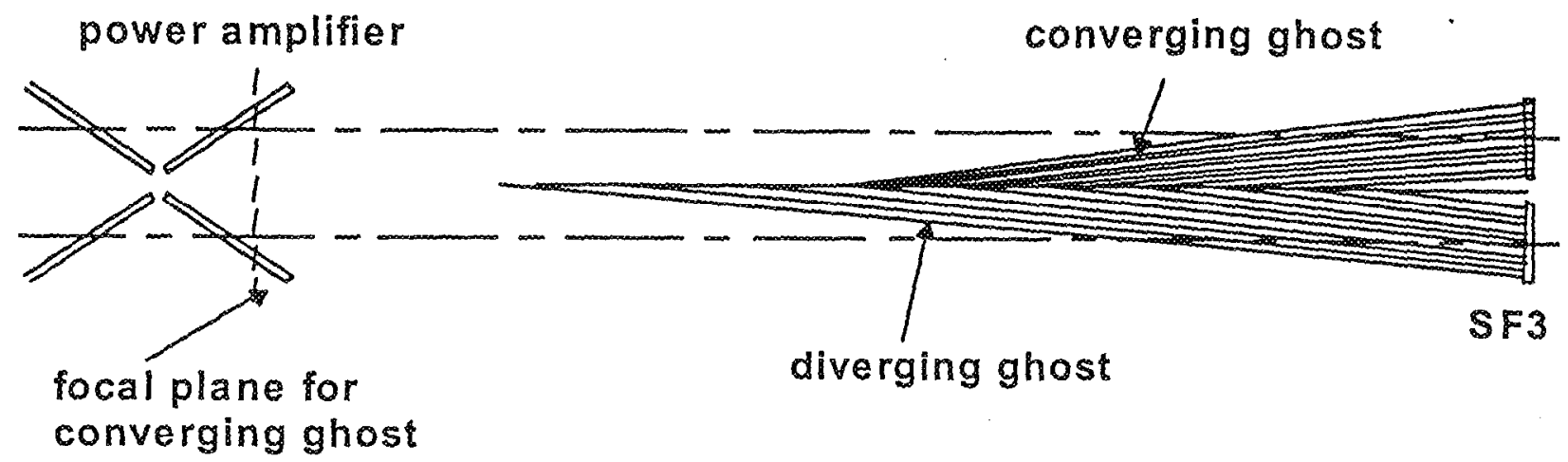

Fig. 3. Shown is a plan view of two adjacent beams between the power amplifier and transport spatial filter lens, SF3. The converging ghost reflections (from side 2) are shown for the beam at the top of the figure; the diverging ghost reflections (from side 1) are shown for the beam at the bottom of the figure. The ghost reflections impinge on absorbing baffles on the beam tube center wall. The focal plane for the converging ghost is about $7 \mathrm{~m}$ from SF3 and would fall in the power amplifier if the lenses were not tilted.

\section{Pockels cell ghost reflections}

Ghost reflections from the Pockels cell, in the main laser cavity, were managed differently. The Pockels cell includes two windows (fused silica) and a crystal (KDP). Reflections from the flat surfaces head back into the spatial filter and are focused by the lens to the same focal plane as the main beam. These reflections are powerful ghosts at focus and must be absorbed by beam dumps before reaching focus.

The ghost management strategy is fairly straightforward: tilt and wedge the elements so that the fluence on beam dumps positioned outside of the beam aperture is reasonable (i.e., $<10 \mathrm{~J} / \mathrm{cm}^{2}$ ). The major considerations were the performance of the $z$-cut KDP crystal when oriented away from normal incidence ( $<10 \mathrm{mrad}$ ) and constraints imposed by the mechanical design of the spatial filter hardware. As it turned out, the mechanical design of the spatial filter beam tubes offered an opportunity for ghost management.

The cavity spatial filter is $23.5 \mathrm{~m}$ long and consists of three vacuum vessels connected by tubes. The end vessels are the interface to the lens mounts. A center vessel provides the mounting platform for the pinhole assemblies. An end vessel is connected to a center vessel by beam tubes for each laser beam. A relatively long section from the end vessel is $440 \mathrm{~mm}$ diameter; a shorter section, connected to the center vessel, is $150 \mathrm{~mm}$ diameter. (These diameters were chosen based on manufacturing costs to match the beam size as it focuses down heading toward the center vessel.) The "step" from the larger tube diameter to the smaller diameter is a convenient location to position the beam dump and is consistent with the restriction to a tilt of less than $10 \mathrm{mrad}$ (a tilt of 6 mrad has been specified). 
It was important during the optical analysis to include the distortion of the switch windows. The interior of the Pockels cell is essentially evacuated; therefore, the windows "bow in." In use; the window surfaces are not plano but have a very long radius of curvature. The paraxial focus for the ghost reflection from SW1 for pass 4 (light traveling left-to-right in Fig. 1) is shifted to the right and from SW2 is shifted to the left (both by $>1 \mathrm{~m}$ ). A raytrace analysis that included the distortion effect and properly modeled the actual beam path through the system was used to determine the expected footprints from each surface for beam passes 3 and 4 . This raytrace data was used to specify the absorber size and location.

\section{Multi-order ghost reflections in the periscope}

The higher-order ${ }^{\dagger}$ ghosts involving the spatial filter lens, SF2, near the Pockels cell required more involved analysis. The analysis techniques included nonsequential raytrace modeling, paraxial raytracing using a spreadsheet and surface tree search to fourth-order, conventional raytrace modeling, and diffraction/propagation modeling.

The first-order ghosts (one bounce) have many higher-order offspring that focus near the Pockels cell. There are two second-order, 36 third-order, and 96 fourth-order ghosts that are worrisome. Two second-order families involve the lens and the end mirror, LM2. They originate at the lens (inner or outer surface), reflect off the cavity end mirror, and finally reflect off the inner surface of the lens. Many fourth-order families involve the Pockels cell surfaces and the lens. They originate at the Pockels cell, reflect off the lens, reflect again off the Pockels cell, and finally refiect off the inner surface of the lens. A significant second-order ghost is shown in Fig. 4.

The vacuum deflection of the lens had to be included to compute the far-field fluences for these ghosts accurately. The ghost focus that is near the switch window would have a wavefront error
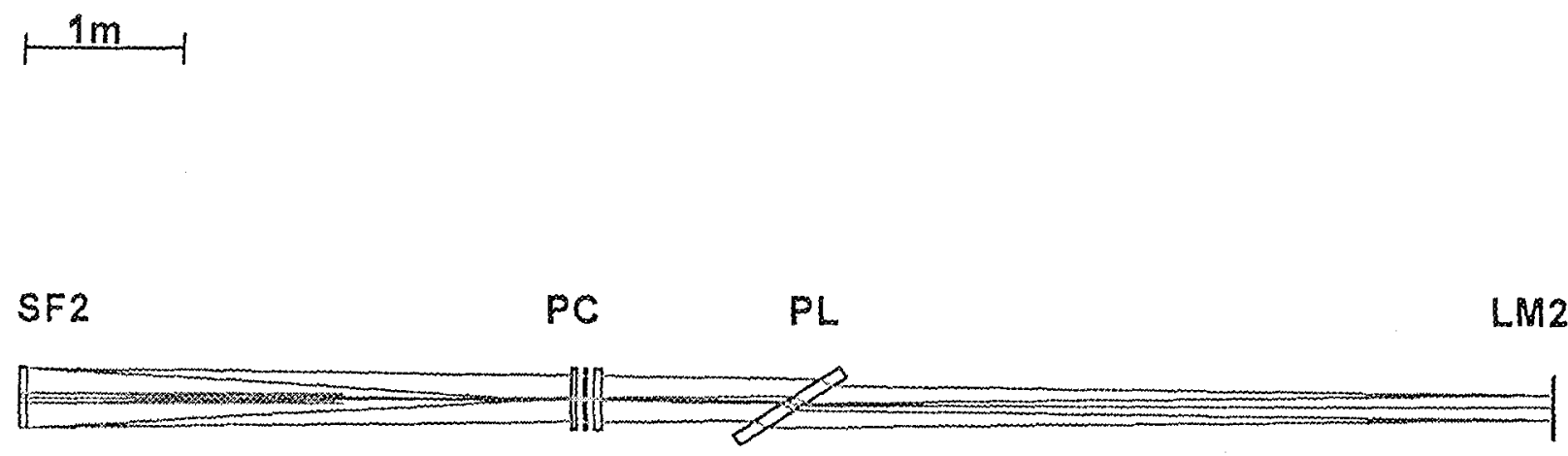

Fig. 4. The ghost path for pass 3 (main laser beam traveling right-to-left) that involves SF2 (inner), LM2, and SF2 (inner) is shown. Only a $66 \mathrm{~mm}$ subaperture of the full beam contributes to the energy at focus (due to clipping at apertures in the system; however, the ghost focuses close to the Pockels cell (PC) elements.

\footnotetext{
'The "order" of a ghost refers to the number of reflections that occur from a nominally anti-reflection coated surface. A third-order ghost, then, has encountered three such surfaces in reflection.
} 
of 9.6 P.V over the full aperture (ghost reflections are usually very highly aberrated); however, only a $66 \mathrm{~mm}$ region contributes to the fluence at focus because of clipping on other apertures in the system. In this case, the distorted lens shape partly corrects the spherical aberration in the ghost reflection. Diffraction propagation calculations estimated the peak fluence to be about $60 \mathrm{~J} / \mathrm{cm}^{2}$.

Given the strength of this ghost and its proximity to optical elements, we studied the uncertainty in location of this ghost due to optical tolerances. For example, because of the change in shape of the spatial filter lens due to vacuum load, there is a $40 \mathrm{~mm}$ shift (paraxial effect) due to the base radius changing. The focal length and radius of curvature tolerances specified gave an uncertainty of $+1.90 \mathrm{~mm}$ on the position of this ghost.

This ghost was identified as a problem with the preliminary design by the nonsequential model and verified by the sequential model. Estimates of the fluence at and near focus were made with the nonsequential code and other diffraction modeling codes. This was identified early enough to implement a relatively inexpensive solution. A change order was issued to the architectural engineering firm to "move" the concrete support pillars upon which structures are mounted by $120 \mathrm{~mm}$; this was much less expensive than a later change order to the building contractor. The increased cavity length affects the end-to-end imaging and propagation negligibly. We did not have to change the lens focal length or shape, tighten the tolerances, or change the design of support structures or opto-mechanical hardware.

\section{Conclusion}

On NIF, the ghost and stray light analysis has been done concurrent with the optical design. Traditionally, this is not the case: the analysis is done after the optical design is essentially complete, and implementation of mitigating measures is often difficult and costly.

Concurrent analysis and design afforded us the opportunity to address problems and concerns in an integrated, systematic manner. The design evolved from conceptual (first-order calculations) to preliminary (raytrace codes) to final (nonsequential modeling). We did not have the "luxury" of completing the optical design and analysis before the mechanical engineers moved to the final design stage. Nevertheless, the concurrent analysis allowed design trade-offs to be investigated while simple, straightforward approaches were still options. This meant that lower cost (projectwide) solutions were implemented.

\section{References}

1. R. English Jr. et al., "Optical system design of the National Ignition Facility," 1998 International Optical Design Conference, paper LFB1.

2. R. Korniski et al., "Spatial filter lens design for the main laser of the National Ignition Facility," 1998 International Optical Design Conference, paper LFB2.

*This work was performed under the auspices of the U.S. Department of Energy by Lawrence Livermore National Laboratory under Contract No. W-7405-Eng-48. 